\title{
Implicit function equation with discontinuous trajectories
}

Yuliya Kaplun, Mykola Perestyuk, and Valeriy Samoylenko 


\title{
IMPLICIT FUNCTION EQUATION WITH DISCONTINUOUS TRAJECTORIES
}

\author{
Yuliya Kaplun, Mykola Perestyuk ${ }^{(1)}$, Valeriy Samoylenko ${ }^{(2)}$ \\ Mechanic and Mathematical Faculty, Kyiv National Taras Shevchenko University \\ Volodymyrska, 64, Kyiv, Ukraine \\ ${ }^{(1)}$ pmo@mechmath.univ.kiev .ua ${ }^{(2)}$ svhr@mechmath.univ.kiev.ua
}

[Received September 18, 2001]

\begin{abstract}
We study an implicit function equation $g(t, x)=0$ with discontinuous trajectories appearing as generating problem in the singular perturbed differential equations with impulsive effects. The existence and periodicity of solutions to the problem are considered.
\end{abstract}

Mathematical Subject Classification: 34K45, 26B10

Keywords: Discontinuous trajectories, implicit function equation, impulsive effects, periodic solution

\section{Introduction}

At present studying of differential equations with impulsive effects [1-5,6] is of great importance. The principal feature of the given equations is that their solutions form the set of power continuum. Such systems are essentially nonlinear and possess a number of specific effects caused by the presence of impulsive actions [6]. Many different problems for systems with impulsive effects written usually by ordinary differential equations, partial differential equations or delay differential equations [7] have been considered so far. At the same time while studying a non-perturbed problem for singularly perturbed differential equations with the impulsive effects [8], the problem of implicit function equations with discontinuous trajectories (with impulsive effects) research appears. In contrast with the case of differential equations with impulsive effects, the corresponding problem for equations can have a countable or even a finite number of solutions.

In this paper we study a system defined by the implicit function equation:

$$
g(t, x)=0
$$

and the condition of impulsive effects:

$$
\left.\Delta x\right|_{t=s_{n}}=x\left(s_{n}+0\right)-x\left(s_{n}-0\right)=I_{n}(x), n \in \mathcal{N} \subset \mathbb{N}
$$

The function $g(t, x)$ is supposed to be continuous in variables $(t, x) \in D=\mathcal{T} \times \mathbb{R}^{1}$, where $\mathcal{T}$ is an open linear connected set from $\mathbb{R}^{1}$, and to have almost everywhere (by Lebesgue measure) the first order continuous derivatives with respect to $t, x$, i.e., 
$g(t, x) \in C_{(t, x)}^{(1,1)}(D)$ almost everywhere. We assume the set $\mathcal{I}=\left\{s_{n}, n \in \mathcal{N}\right\} \subset \mathcal{T}$ to be not empty. Functions $I_{n}(x), n \in \mathcal{N}$, are continuous on $\mathbb{R}^{1}$. Moments of impulsive effects $s_{n} \in \mathcal{I}, n \in \mathcal{N}$, are fixed and additionally a real number $\delta>0$ exists such that $s_{n+1}-s_{n} \geq \delta$ for all $n \in \mathcal{N}$.

\section{Definition of solutions and preliminary notes}

We formulate below some definitions, which will be substantially used in the future.

Definition 2.1. [9] A point $\left(t_{0}, x_{0}\right) \in \mathcal{L}=\{(t, x) \in \bar{D}: g(t, x)=0\}$ is called a critical point of the equation (1.1) any of its deleted neighborhoods $\dot{V}\left(t_{0}, x_{0}\right)$ contains a point from the set $\mathcal{L}$ and either there is not a neighborhood $U$ of the point $\left(t_{0}, x_{0}\right)$ such that $g(t, x)$ is continuously differentiable at every point $(t, x) \in U\left(t_{0}, x_{0}\right)$ or the function $g(t, x)$ is continuously differentiable in some neighborhood $U\left(t_{0}, x_{0}\right)$ but $g_{x}^{\prime}\left(t_{0}, x_{0}\right)=0$.

Definition 2.2. A function $x=x(t), t \in(\alpha, \beta)$, is called a solution to the equation (1.1) if $(t, x(t)) \in D$ for any $t \in(\alpha, \beta)$, the equality $g(t, x(t))=0$ holds and the set $\{(t, x(t)): t \in(\alpha, \beta)\}$ contains no critical points.

Definition 2.3. An isolated solution to the equation (1.1) is a point $\left(t_{0}, x_{0}\right) \in \mathcal{L}$ isolated in the set $\mathcal{L}$.

Definition 2.4. The point $\left(t_{*}, x_{*}\right) \in \mathcal{L}$ that is neither a critical point nor an isolated solution is called a regular point of the equation (1.1) .

Under the conditions imposed above, for any point $\left(t_{0}, x_{0}\right) \in \mathcal{L} \backslash \partial \mathcal{L}[9]$ there is a continuously differentiable solution $x(t)$ to the equation (1.1) defined on the maximal interval $\left(\omega_{-}, \omega_{+}\right)$such that $x\left(t_{0}\right)=x_{0}$.

Further it would be relevant to mention the following definitions:

Definition 2.5. A point $\left(t_{*}, x_{*}\right)$ is called a left finite critical point of the equation (1.1) if for some solution $x=\varphi(t)$ of the equation (1.1) limit $\lim _{t \rightarrow t_{*}-0} \varphi(t)=x_{*}$ exists and $\left|x_{*}\right|<+\infty$. In the case $\left|x_{*}\right|=+\infty$ the point $\left(t_{*}, x_{*}\right)$ is called a left infinite critical point of the equation (1.1) .

Definition 2.6. A piecewise continuous function $x=x(t), t \in(\alpha, \beta)$, is called a bounded solution to the problem (1.1), (1.2), if:

(1) function $x=x(t)$ satisfies the equation (1.1) for all $t \in(\alpha, \beta) \backslash \mathcal{I}$;

(2) the set $\{(t, x(t)): t \in(\alpha, \beta) \backslash \mathcal{I}\}$ contains no critical points of equation (1.1);

(3) function $x(t)$ is left continuous at points $t=s_{n}$ and satisfies condition (1.2).

Let $x=\varphi(t)$ be a solution to equation (1.1) defined on the maximal interval $\left(\alpha, t_{*}\right)$. It easy to show that the point $\left(t_{*}, \varphi\left(t_{*}\right)\right)$, where $\varphi\left(t_{*}\right)=\lim _{t \rightarrow t_{*}-0} \varphi(t)$, is a critical point of equation (1.1) . 
Definition 2.7. A piecewise continuous function $x=x(t):(\alpha, \beta) \rightarrow \overline{\mathbb{R}}^{1}$ is called a solution to the problem (1.1), (1.2), if:

(1) function $x=x(t)$ satisfies the equation (1.1) for all $t \in(\alpha, \beta) \backslash \mathcal{I}$;

(2) the set $\{(t, x(t)): t \in(\alpha, \beta) \backslash \mathcal{I}\}$ contains no critical points of the equation (1.1) ;

(3a) if the point $s \in \mathcal{I} \cap(\alpha, \beta)$ and $|x(s-0)|<+\infty$, then there exists a neighborhood $U(s)$ such that function $x=x(t), t \in U$, is a bounded solution to the problem (1.1), (1.2) in the sense of the definition 2.6;

(3b) if the point $s \in \mathcal{I} \cap(\alpha, \beta)$ and $|x(s-0)|=+\infty$, then the point $(s, \infty)$ is a left and right infinite critical point of the equation (1.1) simultaneously.

It should be noted here that if a solution to the problem $(1.1),(1.2)$ is defined in both the left and right neighborhoods of the point $s_{n} \in \mathcal{I}$, which coincides with $t$-coordinate of an infinite critical point $\left(s_{n}, \infty\right)$, then it necessarily follows the point $\left(s_{n}, \infty\right)$ is neither right continuous nor left continuous. By another way if the point $\left(s_{n}, x_{n}\right)$ is a left infinite critical point then it is also a right infinite critical point.

If the limit $\lim _{t \rightarrow t_{*}-0} \varphi(t)$ does not exist, then there is a sequence $\left\{t_{k}, k \geq 1\right\}$ such that $t_{k} \in\left(\alpha, t_{*}\right), t_{k} \rightarrow t_{*}$ as $k \rightarrow \infty$ and limit $\lim _{k \rightarrow \infty} \varphi\left(t_{k}\right)=: \varphi\left[\left\{t_{k}\right\}\right]$ exists. The set of all these points $\varphi\left[\left\{t_{k}\right\}\right]$ forms a linear connected set $[a, b] \subset \overline{\mathbb{R}}^{1}$. In this case a point $\left(t_{*}, a_{*}\right)$ where $a_{*} \in[a, b]$ is called a left continual critical point of the equation (1.1) .

Further we suppose the equation (1.1) has no continual critical points. Analogously to definition 2.5, it is possible to introduce notions of a right finite, a right infinite and a right continual critical point of the equation (1.1) .

It should be noted that point $\left(t_{*}, x_{*}\right)$ can be both a left critical point for a solution $\varphi_{1}(t), t \in\left(\alpha, t_{*}\right)$, and a right critical point for another solution $\varphi_{2}(t), t \in\left(t_{*}, \beta\right)$.

In the case the point $\left(t_{*}, x_{*}\right)$ is a left critical point of the equation (1.1), there exists a solution $\varphi(t)$ to the equation (1.1) such that $\lim _{n \rightarrow \infty} \varphi\left(t_{n}\right)=x_{*}$ exists for any monotone increasing sequence $\left\{t_{n}, n \leq 1\right\} \subset\left(\alpha, t_{*}\right)^{n \rightarrow \infty}: t_{n} \rightarrow t_{*}$ as $n \rightarrow \infty$ and $g\left(t_{n}, \varphi\left(t_{n}\right)\right)=0$. Using this property, it is possible to clarify whether or not point $\left(t_{*}, x_{*}\right)$ is a left critical point for some solution to the equation (1.1). Namely, if a sequence $\left\{\left(t_{n}, x_{n}\right), n \geq 1\right\}: g\left(t_{n}, x_{n}\right)=0$ and $\left(t_{n}, x_{n}\right) \rightarrow\left(t_{*}-0, x_{*}\right)$ as $n \rightarrow \infty$ exists, then the point $\left(t_{*}, x_{*}\right)$ is a left critical point of the equation (1.1). Otherwise the point $\left(t_{*}, x_{*}\right)$ is a right critical point.

Remark 2.1. Any critical point can be a point of non-uniqueness [10] of the solutions to the equation (1.1) . 


\section{Necessary condition of existence of a solution}

Assuming the equation (1.1) has no isolated solution, let us study conditions of existence of a solution to the problem (1.1), (1.2).

Lemma 3.1. Let function $x=x(t), t \in \mathcal{T}$, be a solution to the problem (1.1), (1.2). Then:

(1) if a point $\left(s_{n}, x\left(s_{n}-0\right)\right)$ is either a regular point or a left critical point of the equation (1.1), then point $\left(s_{n}, x\left(s_{n}+0\right)\right)$ is either a regular or a right finite critical point of the equation (1.1);

(2) a point $\left(s_{n}, x\left(s_{n}-0\right)\right)$ is a left infinite critical point of the equation (1.1), iff $\left(s_{n}, x\left(s_{n}+0\right)\right)$ is a right infinite critical point of the equation (1.1). .

Proof of the lemma follows from functions $I_{n}(x)$ continuity.

Remark 3.1. Let us suppose:

(1) a point $\left(s_{n}, x\left(s_{n}-0\right)\right)$, a solution $x(t)$ to the problem $(1.1),(1.2)$ passes through, is a left infinite critical point of the equation (1.1) ;

(2) solution $x(t)$ is defined on an interval, the opened part of which contains point $s_{n}$.

Then point $\left(s_{n}, x\left(s_{n}+0\right)\right)$ is a right infinite critical point of the equation (1.1). In addition, the value $I_{n}\left(x_{n}\right)=\lim _{t \rightarrow s_{n}-0} I_{n}(x(t))$ is considered to be finite.

Theorem 3.1. (necessary condition of existence of a solution to the problem (1.1), (1.2) ). If the problem (1.1), (1.2) has a solution $x(t), t \in \mathcal{T}$, then there are points $x_{n} \in \overline{\mathbb{R}}^{1}, n \in \mathcal{N}$, such that $\left(s_{n}, x_{n}\right) \in \mathcal{L}, n \in \mathcal{N}$, and one of the following conditions takes place:

(i) if $x_{n} \in \mathbb{R}^{1}$ then equality $g\left(s_{n}, x_{n}+I_{n}\left(x_{n}\right)\right)=0$ holds;

(ii) if $x_{n} \in \overline{\mathbb{R}}^{1} \backslash \mathbb{R}^{1}$ then point $\left(s_{n}, x\left(s_{n}-0\right)\right)$ is a left infinite critical point and point $\left(s_{n}, x\left(s_{n}+0\right)\right)$ is a right infinite critical point of the equation (1.1).

Proof. To prove the theorem it is enough to verify its statement for a point where solution $x(t), t \in \mathcal{T}$, has discontinuity.

Let $x=x(t)$ be a solution to the problem (1.1), (1.2), defined on $\mathcal{T}$. In accordance with the definition of a solution to the problem $(1.1),(1.2)$ function $x(t)$ is continuously differentiable on the set $\mathcal{T}$, excepting points of impulsive effects where it is only left continuous. 
Let $s_{n_{0}} \in \mathcal{T}$ be an arbitrary point from the set $\mathcal{I}$. The case when the point $\left(s_{n_{0}}, x\left(s_{n_{0}}-0\right)\right)$ is a left infinite critical point or the point $\left(s_{n_{0}}, x\left(s_{n_{0}}+0\right)\right)$ is a right infinite critical one of the equation (1.1) is mentioned above in remark 3.1 ; thus we suppose the solution $x(t)$ to have a discontinuity of the first kind at the point $s_{n_{0}}$.

Let us consider set $\left\{\left(s_{n_{0}}, x_{n}\right), n \in \mathcal{N}\right\}$, where $x_{n}=\lim _{t_{k} \rightarrow s_{n}-0} x(t)$ as $t_{k} \rightarrow s_{n_{0}}$ and $t_{k}<s_{n_{0}}$. Since the given solution $x(t)$ is defined on the whole set $\mathcal{T}$, and none of points $\left(s_{n_{0}}, x_{n}\right)$ is a continual critical point of the equation (1.1), then for any $n$ set $\left\{\left(s_{n_{0}}, x_{n}\right), n \in \mathcal{N}\right\}$ can contain the only point.

Assuming the correspondence $x_{n_{0}}$ to $s_{n_{0}}$ for any $n_{0} \in \mathcal{N}$ and taking into account that function $x(t)$ satisfies the equation (1.1), we infer the set $\left\{\left(s_{n_{0}}, x_{n}\right), n \in \mathcal{N}\right\}$ to be a subset of the set $\mathcal{L}$. Hence the equality $g\left(s_{n}, x_{n}+I_{n}\left(x_{n}\right)\right)=0$ is true for any $n \in \mathcal{N}$ and some set $\left\{x_{n}, n \in \mathcal{N}\right\}$ that completes the proof of the theorem.

Theorem 3.2. Let set $\mathcal{T} \times^{-1}$ contain only finite critical points of the equation (1.1); the functions $I_{n}(x), n \in \mathcal{N}$ are bounded. If the problem (1.1), (1.2) has the solution defined on the interval $(\alpha, \beta)$, then this solution is necessarily bounded on every part $[a, b] \subset(\alpha, \beta)$.

Proof. Let a function $x(t)$ satisfying the problem (1.1), (1.2) be defined on interval $(\alpha, \beta)$ and unbounded on a subinterval $\left[a_{1}, b_{1}\right] \subset(\alpha, \beta)$. In this case there is a point $t_{0} \in\left[a_{1}, b_{1}\right]$ such that $\lim _{t \rightarrow t_{0}} x(t)=\infty$. In accordance with definition 2.1 , it is possible to deduce that the equation (1.1) has a critical point $\left(t_{0}, \infty\right) \in \mathcal{T} \times \overline{\mathbb{R}}^{1}$. The last assumption contradicts the supposition of the theorem and concludes the proof.

\section{Necessary condition of existence of a periodic solution}

It should be mentioned here that analogy of the necessary condition of the existence of a periodic solution to a differential equation with impulsive effects $[2,5]$ also holds in the case of the problem (1.1), (1.2). Namely the following theorem is true:

Theorem 4.1. (necessary condition of existence of a periodic solution to the problem (1.1), (1.2)). If the problem (1.1), (1.2) has a T-periodic solution defined on the whole set $\mathcal{T}$, then there are points $x_{n} \in \overline{\mathbb{R}}^{1}, n \in \mathcal{N}$ and a natural number $m \in \mathbb{N}$ such that $\left\{\left(s_{n}, x_{n}\right), n \in \mathcal{N}\right\} \subset \mathcal{L}$ and for any $n \in \mathcal{N}$ the following conditions are fulfilled:

$$
I_{n+m}\left(x_{n}\right)=I_{n}\left(x_{n}\right), \quad s_{n+m}=s_{n}+T, \quad k \in \mathcal{N},
$$

provided that $s_{n+m} \in \mathcal{T}$.

Proof. Let function $\varphi(t)$ be a periodic solution to the problem $(1.1),(1.2)$ and $(\alpha, \beta) \subset$ $\mathcal{T}$ be an interval of its definition. Then we evidently have $\beta-\alpha>T$.

At first we shall show that if a point $s_{n_{0}} \in(\alpha, \beta)$ belongs to the set $\mathcal{I}$ and interval $(\alpha, \beta)$ contains at least one of the points $s_{+}=s_{n_{0}}+T$ or $s_{-}=s_{n_{0}}-T$, then there is 
a natural $m \in \mathbb{N}$ such that for any $n \in \mathcal{N}$ we have $I_{n+m}\left(x_{n}\right)=I_{n}\left(x_{n}\right), n \in \mathcal{N}$ and $s_{n+m}=s_{n}+T$ having supposed $s_{+} \in(\alpha, \beta)$ for determination.

Evidently, inclusion $s_{+} \in(\alpha, \beta)$ follows $s_{+} \in \mathcal{I}$. Otherwise, assuming $s_{+}$to be not a point of an impulsive effect, we can conclude that function $\varphi(t)$ as a solution to the problem (1.1), (1.2)is continuous at $s_{+}$. Hence due to the periodicity condition of theorem 4.1, function $\varphi(t)$ has to be continuous at $s_{n_{0}}=s_{+}-T$, which is impossible.

Thus, if $s_{n_{0}} \in \mathcal{I}$ and $s_{+}=s_{n_{0}}+T \in(\alpha, \beta)$, then $s_{+} \in \mathcal{I}$. As $s_{n_{0}}$ is an arbitrary point, then we can infer that there is a natural $m \in \mathbb{N}$ such that $s_{n+m}=s_{n}+T \in \mathcal{I}$ for any $n \in \mathcal{N}$ provided that $s_{n+m} \in(\alpha, \beta)$. In particular, $m=n_{1}-n_{0}$, where we have denoted $s_{n_{1}}=s_{+}=s_{n_{0}}+T$. We suppose the number $m \in \mathbb{N}$ to be the least such natural number.

Accordingly to theorem 3.1, there follows the existence of set $\left\{x_{n}, n \in \mathcal{N}\right\}$ such that $g\left(s_{n}, x_{n}+I_{n}\left(x_{n}\right)\right)=0$ for any $n \in \mathcal{N}$. Taking the condition of periodicity of the solution $x=\varphi(t)$ in the form: $\varphi(t) \equiv \varphi(t+T) \forall t \in\left(\left(s_{n_{-1}}, s_{n_{1}}\right) \backslash\left\{s_{n_{0}}\right\}\right) \cap \mathcal{T}$, where $s_{n_{1}}=s_{n_{0}}+T, s_{n_{-1}}=s_{n_{0}}-T$, we get the following relationships:

$$
\begin{gathered}
I_{n_{0}}\left(x_{n_{0}}\right)=\varphi\left(s_{n_{0}}+T+0\right)-\varphi\left(s_{n_{0}}+T-0\right)= \\
=\varphi\left(s_{n_{0}+m}+0\right)-\varphi\left(s_{n_{0}+m}-0\right)= \\
=x_{n_{0}+m}+I_{n_{0}+m}\left(x_{n_{0}+m}\right)-x_{n_{0}+m}=I_{n_{0}+m}\left(x_{n_{0}+m}\right)=I_{n_{0}+m}\left(x_{n_{0}}\right) .
\end{gathered}
$$

Thus theorem 4.1 is proved.

\section{Criterion of existence of a solution}

The maximal interval size of definition of a solution to the problem (1.1), (1.2) depends on the existence of critical points of the equation (1.1) in the set $\mathcal{T} \times \overline{\mathbb{R}}^{1}$. We pursue proving the following theorem on necessary and sufficient conditions of the existence of a solution to the problem (1.1), (1.2).

Theorem 5.1. (criterion of existence of a solution to the problem (1.1), (1.2)). Let set $\mathcal{T} \times \overline{\mathbb{R}}^{1}$ contain no critical points of equation (1.1). Then the problem (1.1), (1.2) has a bounded solution defined on the whole set $\mathcal{T}$ iff there are points $x_{n} \in \mathbb{R}^{1}, n \in \mathcal{N}$ such that $\left\{\left(s_{n}, x_{n}\right), n \in \mathcal{N}\right\} \subset \mathcal{L}$ and condition $g\left(s_{n}, x_{n}+I_{n}\left(x_{n}\right)\right)=0$ holds for all $n \in \mathcal{N}$ and, additionally, the following condition takes place: for any $n \in \mathcal{N}$ there is a domain $G_{n} \subset \mathcal{T} \times \mathbb{R}^{1}$ such that

a) points $\left(s_{n}, x_{n}+I_{n}\left(x_{n}\right)\right),\left(s_{n+1}, x_{n+1}\right)$ belong to the domain $G_{n}$ provided that $s_{n}, s_{n+1} \in \mathcal{I}$

b) for any $t_{*} \in\left(s_{n}, s_{n+1}\right)$ the equation $g\left(t_{*}, x\right)=0$ has the only solution $x=x_{*}$ such that $\left(t_{*}, x_{*}\right) \in G_{n}$. 
Proof. At first we note that the necessity of conditions of the theorem follows from theorem 3.1. Indeed, let function $x^{*}(t)$ defined on the whole set $\mathcal{T}$ be a solution to the problem (1.1), (1.2). In accordance with the definition 2.6, function $x^{*}(t)$ is continuously differentiable with respect to $t \in \mathcal{T}$ everywhere, excepting points of impulsive effects $s_{n} \in \mathcal{I}, n \in \mathcal{N}$ ordered by the following way $s_{1}<s_{2}<\ldots$.

Consider sets $\mathcal{T}_{k} \subset \mathcal{T}, k \in \mathcal{N}$ to have the following form: $\mathcal{T}_{1}=\left\{t \in \mathcal{T}: t \leq s_{1}\right\}$, $\mathcal{T}_{2}=\left\{t \in \mathcal{T} \backslash \mathcal{T}_{1}: t \leq s_{2}\right\}$ if $s_{2} \in \mathcal{I}, \ldots, \mathcal{T}_{n}=\left\{t \in \mathcal{T} \backslash \bigcup_{k=1}^{n-1} \mathcal{T}_{k}: t \leq s_{n}\right\}$ if $s_{n} \in \mathcal{I}$

Since set $\mathcal{I}$ is not empty, then there are at least two sets $\mathcal{T}_{1}$ and $\mathcal{T}_{2}$. Let $x_{n}(t)=$ $x^{*}(t), t \in \mathcal{T}_{n}$, for all $n \in \mathcal{N}$. We denote

$$
x_{n}=\lim _{t \rightarrow s_{n}-0} x^{*}(t)
$$

Evidently the set of points $\left\{\left(s_{n}, x_{n}\right), n \in \mathcal{N}\right\}$ is a subset of $\mathcal{L}$ and accordingly to the necessary condition of existence of a solution, i.e., the equality $g\left(s_{n}, x_{n}+I_{n}\left(x_{n}\right)\right)=0$ is true. Necessity is proved.

Let us prove the sufficiency. Assume points $x_{n} \in \mathbb{R}^{1}, n \in \mathcal{N}$ exist. We prove that the problem (1.1), (1.2) has a solution defined on the whole set $\mathcal{T}$. Again we consider subsets $\mathcal{T}_{n} \subset \mathcal{T}, n \in \mathcal{N}$ being devided by the points of impulsive effects $s_{n} \in \mathcal{I}$. Since point $\left(s_{1}, x_{1}\right) \in \mathcal{L}$ is not a critical point of the equation (1.1), then in accordance with theorem [9] on a global existence of a solution to the equation (1.1), there is the only continuously differentiable function $x=x_{1}(t)$ defined on the whole set $\mathcal{T}$ such that $x_{1}\left(s_{1}\right)=x_{1}$. Let $x^{*}(t)$ be a solution to the problem (1.1), (1.2). As before, we define $x_{1}(t)$ as restriction of solution $x^{*}(t)$ on the set $\mathcal{T}_{1}$, i.e., $x^{*}(t)=x_{1}(t)$ for any $t \in \mathcal{T}_{1}$.

Let us construct a right continuation of the solution $x_{1}(t)$. It can be fulfilled because point $\left(s_{1}, x_{1}+I_{1}\left(x_{1}\right)\right) \in \mathcal{L}$ is not a critical point of the equation (1.1). Following the aforecited argument, we are able to affirm the existence of the only continuously differentiable function defined on the whole set $\mathcal{T}$ such that $x_{2}\left(s_{1}\right)=$ $x_{1}+I_{1}\left(x_{1}\right)$. We consider $x_{2}(t), t \in \mathcal{T}_{2}$, as restriction of the solution $x^{*}(t)$ on the set $\mathcal{T}_{2}$, i.e., $x^{*}(t)=x_{2}(t)$ for any $t \in \mathcal{T}_{2}$.

If $s_{2} \notin \mathcal{I}$, i.e., the set $\mathcal{I}$ of points of impulsive effect contains the only point, then function $x^{*}(t)$ is defined on the whole set $\mathcal{T}$. Otherwise we repeat the aforementioned procedure.

So for any $n \in \mathcal{N}$ it is possible to build function $x_{n}(t)$ satisfying both the equation (1.1) for any $t \in \mathcal{T}$ and condition (1.2) at $t=s_{n}$. In addition, its restriction will coincide with the solution $x^{*}(t)$ on the interval $\left(s_{n-1}, s_{n}\right]$.

If the whole set $\mathcal{T}$ is right bounded or the set $\mathcal{I}$ contains only a finite number of points of impulsive effects, then by means of finite steps we are able to construct the solution $x^{*}(t)$ defined on the whole set $\mathcal{T}$. Otherwise, when $\mathcal{T}$ is right unbounded, due to both theorem [9] on global existence of a solution to the equation (1.1) and condition $s_{n+1}>s_{n}+\delta, n \in \mathcal{N}$, we are able to find $x^{*}(t)$ for any $t \in \mathcal{T}$ by a finite number of steps. 
It means that the problem $(1.1),(1.2)$ has a solution defined on the whole set $\mathcal{T}$ that proves the theorem 5.1 .

Let us consider now the case when set $\mathcal{T} \times \overline{\mathbb{R}}^{1}$ contains some critical points of the equation (1.1). Let us draw lines through the critical points being paralleled to axis $O X$ and assume that number of such lines is at most countable. We denote these lines as $t=\tau_{m}, m \in B \subset \mathbb{N}$.

At first we consider the case when the set $\mathcal{T}$ contains only finite critical points.

Theorem 5.2. Let the critical points of the equation (1.1) belonging to the set $\mathcal{T} \times \overline{\mathbb{R}}^{1}$ be only isolated finite critical points which are necessarily being elements of the set $\mathcal{I}$. Then the problem (1.1), (1.2) has a solution defined on the whole set $\mathcal{T}$ iff the following conditions are fulfilled:

1) there is a point $(\bar{t}, \bar{x}) \in \mathcal{L}$ such that $\bar{t}<s_{1}$;

2) there are points $x_{n} \in \mathbb{R}^{1}, n \in \mathcal{N}$ such that condition i) of the theorem 3.1 holds;

3) for any $n \in \mathcal{N}_{0}=\mathcal{N} \cup\{0\}$ there is a domain $G_{n}$ such that $(\bar{t}, \bar{x}),\left(s_{1}, x_{1}\right) \in \partial G_{0}$ and points $\left(s_{n}, x_{n}+I_{n}\left(x_{n}\right)\right),\left(s_{n+1}, x_{n+1}\right) \in \partial G_{n}$ if $s_{n+1} \in \mathcal{I}$;

4) for any $t_{*} \in\left(s_{n}, s_{n+1}\right)$ the equation $g\left(t_{*}, x\right)=0$ has the only solution $x=x_{*}$ such that $\left(t_{*}, x_{*}\right) \in G_{n}$.

Proof. Let us start proving the necessity of conditions of theorem 5.2. We denote the finite critical points of equation (1.1) belonging to the set $\mathcal{T} \times \overline{\mathbb{R}}^{1}$ by the following $\left\{\left(\tau_{m}, \kappa_{m_{l}}\right), m \in B, l \in \mathbb{Z}\right\}$, where in accordance with assumptions of theorem 5.2 $\left\{\tau_{m}, m \in B\right\} \subset \mathcal{I}$.

Let the function $x^{*}(t)$ defined on the whole set $\mathcal{T}$ be a solution to the problem (1.1), (1.2). Evidently, there is a point $(\bar{t}, \bar{x}) \in \mathcal{L}, \bar{t}<s_{1}$, since we can put $\bar{x}=x^{*}(\bar{t})$ for any $\bar{t} \in \mathcal{T}$ such that $\bar{t}<s_{1}$. Thus, the first condition holds.

By means of the argument used to prove theorem 5.1, we can show the existence of points $x_{n} \in \mathbb{R}, n \in \mathcal{N}$ such that $\left\{\left(s_{n}, x_{n}\right), n \in \mathcal{N}\right\} \subset \mathcal{L}$ and for every point $\left(s_{n}, x_{n}\right)$ the condition i) of theorem 3.1 holds.

Since the set $\left(s_{n}, s_{n+1}\right) \times \overline{\mathbb{R}}^{1}$ contains no critical points, there are not two different solutions to the equation (1.1) passing through the same point belonging to set $\left(s_{n}, s_{n+1}\right) \times \overline{\mathbb{R}}^{1}$ because such a point would be a critical point of the equation (1.1).

Taking into account that all critical points of equation (1.1) belonging to $\mathcal{T} \times \mathbb{R}^{1}$ are isolated, we conclude that for any $n \in \mathcal{N}$ there is a domain $G_{n}$ such that condition 3 ) is satisfied, for we can consider an open linear connected set being a small enough neighborhood of the function $x_{n}(t), t \in \mathcal{T}_{n}, n \in \mathbb{N}$, graph as the set $G_{n}, n \in \mathbb{N}_{0}$. Additionally, condition 4) would be fulfilled.

Thus the necessity is proved. 
Having assumed conditions 1) - 4) of theorem 5.2 to hold we proceed to prove sufficiency. Let us show that the problem (1.1), (1.2) has a solution defined on the whole set $\mathcal{T}$. Since a point $(\bar{t}, \bar{x})$ belongs to the set $\mathcal{L}$, then due to theorem [9] on the existence of a global solution to equation (1.1) there is a continuously differentiable function $x_{1}(t)$ defined at least on the set $\mathcal{T}_{1}$ such that $x_{1}(\bar{t})=\bar{x}$.

The solution $x_{1}(t)$ admits right-side continuation. Indeed, if $\left(s_{1}, x_{1}+I_{1}\left(x_{1}\right)\right)$ is a regular point of equation (1.1), then in accordance with theorem 5.1 this solution can be extended to the next interval $\left(s_{1}, s_{2}\right]$; if $\left(s_{1}, x_{1}+I_{1}\left(x_{1}\right)\right)$ is a critical point of equation (1.1), then from condition 3 of the theorem it follows that this point is a left finite critical one for equation (1.1) and, consequently, equation (1.1) has a solution $x_{2}(t)$ defined at least on the set $\mathcal{T}_{2}$ satisfying the condition $x_{2}\left(s_{1}+0\right)=x_{1}+I_{1}\left(x_{1}\right)$, or, in other words, "entering" into this critical point.

If set $\mathcal{I}$ contains the only point $s_{1}$, then function $x_{2}(t)$ is defined on set $\mathcal{T}_{2}=\mathcal{T} \backslash \mathcal{T}_{1}$ and as a result function $x^{*}(t)$, composed on functions $x_{1}(t), x_{2}(t)$, is defined on the whole set $\mathcal{T}$, satisfying the equation (1.1) and condition (1.2), i.e., it is a solution to the problem (1.1), (1.2).

If $s_{2} \in \mathcal{I}$, then the function $x_{2}(t)$ is defined on set $\mathcal{T}_{2}$ and we come across a problem of $x_{2}(t)$ continuation on the next set $\mathcal{T}_{3}$ that we can solve using the speculations given above. Thus, for any point $s_{n} \in \mathcal{I}$ it is possible to construct function $x_{n}(t)$ defined on set $\mathcal{T}_{n}$.

If set $\mathcal{T}$ is right bounded or contains only a finite number of its elements, then by a finite number of steps we are able to construct solution $x^{*}(t)$ defined on the whole set $\mathcal{T}$. Otherwise, when set $\mathcal{T}$ is right unbounded, then due to both theorem [9] on the global existence of a solution to equation (1.1) and condition $s_{n+1}>s_{n}+\delta$, $n \in \mathcal{N}$ for any $t>s_{1}$, the value $x^{*}(t)$ can be found by means of a finite number of the aforementioned steps. Thus theorem 5.2 is proved.

Theorem 5.3. Let set $\mathcal{T} \times \overline{\mathbb{R}}^{1}$ contain only infinite critical points of the equation (1.1) which form set $\left\{\left(\tau_{k}, \infty\right), k \in B \subset\right\}$ where $\tau_{k} \in \mathcal{I}$ for all $k \in B$. Then the problem (1.1), (1.2) has a solution defined on the whole set $\mathcal{T}$ iff the following conditions take place:

1) there is a point $(\bar{t}, \bar{x}) \in \mathcal{L}$ such that $\bar{t}<s_{1}$;

2) there are points $x_{n} \in \overline{\mathbb{R}}^{1}, n \in \mathcal{N}$ such that:

a) $\left\{\left(s_{n}, x_{n}\right), n \in \mathcal{N}\right\} \subset \mathcal{L}$;

b) condition i) or ii) of theorem 3.1 holds;

3) for any $n \in \mathcal{N} \cup\{0\}$ there exists a domain $G_{n} \subset \mathcal{T} \times \mathbb{R}^{1}$ such that:

a) set $G_{0} \cup\left\{\left(s_{1}, x\right):-\infty \leq x \leq+\infty\right\}$ includes points $(\bar{t}, \bar{x})$ and $\left(s_{1}, x_{1}\right)$;

b) for any $n \in \mathcal{N}$ set $G_{n} \cup\left\{\left(s_{n}, x\right):-\infty \leq x \leq+\infty\right\} \cup\left\{\left(s_{n+1}, x\right):-\infty \leq x \leq\right.$ $+\infty\}$ includes points $\left(s_{n}, x_{n}+I_{n}\left(x_{n}\right)\right),\left(s_{n+1}, x_{n+1}\right)$ provided that $s_{n+1} \in \mathcal{I}$; 
4) for all $n \in \mathcal{N}$ and any $t_{*} \in\left(s_{n}, s_{n+1}\right)$ the equation $g\left(t_{*}, x\right)=0$ has the only solution $x=x_{*}$ such that $\left(t_{*}, x_{*}\right) \in G_{n}$.

Proof. Necessity of the conditions is the first item to be proved. Let function $x^{*}(t)$ defined on the whole set $\mathcal{T}$ be a solution to the problem (1.1), (1.2). Set $\mathcal{I}$, being not empty according to the assumption, contains at least one element $s_{1}$. Thus there is a point $\bar{t} \in \mathcal{T}$ such that $\bar{t}<s_{1}$ and value $\bar{x}=x^{*}(\bar{t})$ is defined. So, condition 1$)$ holds.

In accordance with definition 2.6, function $x^{*}(t)$ is continuously differentiable for all $t \in \mathcal{T}$ excepting points of impulsive effects $s_{n} \in \mathcal{I}$. For any $n \in \mathcal{N}$ such that $s_{n} \in \mathcal{I}$ let denote $x_{n}(t)=x^{*}(t)$ for $s_{n}<t \leq s_{n+1} ; x_{0}(t)=x^{*}(t)$ for $t \in \mathcal{T} \cap\left(-\infty, s_{1}\right]$.

Similarly as in the proof of theorem 5.1 , we put $x_{n}=\lim _{t \rightarrow s_{n}-0} x^{*}(t)$ for all $n \in \mathcal{N}$.

Obviously, the set of points $\left\{\left(s_{n}, x_{n}\right), n \in \mathcal{N}\right\}$ is a subset of $\mathcal{L}$; hence, according to theorem 3.1, the necessary conditions of existence of a solution are fulfilled.

Let us consider an interval $\left(s_{n}, s_{n+1}\right]$, where $s_{n}, s_{n+1} \in \mathcal{I}$. If $\left(s_{n+1}, x_{n+1}\right)$ is a critical point for the equation (1.1), then refereing to the input conditions of theorem 5.3 , we find this point to be a right infinite critical point of solution $x_{n}(t)=x^{*}(t)$, $t \in\left(s_{n}, s_{n+1}\right]$ and as a consequence, this point is a left infinite critical point of solution $x_{n+1}(t)=x^{*}(t), t \in\left(s_{n+1}, s_{n+2}\right]$.

In conclusion we note that for any $n \in \mathcal{N} \cup\{0\}$ domain $G_{n}$ can be chosen as a small enough neighborhood of graph of the function $x_{n}(t), t \in\left(s_{n}, s_{n+1}\right]$, such that conditions 3) and 4) have to take place. Thus, the necessity has been demonstrated.

Now sufficiency is to be proved. Let conditions 1) - 4) be right. By direct method of constructing a function we shall show that the problem (1.1), (1.2) has a solution defined on the whole set $\mathcal{T}$. Let us consider subsets on which the set $\mathcal{T}$ is divided by points of impulsive effects $s_{n} \in \mathcal{I}$ starting from interval $\left(-\infty, s_{1}\right] \cap \mathcal{T}$. As point $(\bar{t}, \bar{x})$ belongs to set $\mathcal{L}$ then according to theorem [9] on the existence of a global solution to the equation (1.1), there is a continuously differentiable function $x_{1}(t)$ defined at least on interval $\mathcal{T} \cap\left(-\infty, s_{1}\right)$ such that $x_{1}(\bar{t})=\bar{x}$. Denoting solution to the problem (1.1), (1.2) defined on the set $\mathcal{T}$ as $x^{*}(t)$, we obviously get $x^{*}(t)=x_{1}(t)$ for all $t \in \mathcal{T} \cap\left(-\infty, s_{1}\right)$. In addition, $x_{1}\left(s_{1}\right)=x_{1}$.

Let us build the right prolongation of function $x_{1}(t)$. If $\left(s_{1}, x_{1}\right)$ is a regular point of the equation (1.1), then, due to theorem 5.1, solution to the problem (1.1), (1.2) can be right-side extended either on interval $\left(s_{1}, s_{2}\right]$ if $s_{2} \in \mathcal{I}$ or set $\mathcal{T} \backslash\left(-\infty, s_{1}\right]$ if $s_{2} \notin \mathcal{I}$. Otherwise, if $\left(s_{1}, x_{1}\right)$ is a critical point of the equation (1.1), then it is only an infinite critical one. Thus relying on definition 2.7, we get solution $x_{2}(t)$ to the problem (1.1), (1.2) defined either on interval $\left(s_{1}, s_{2}\right]$ if $s_{2} \in \mathcal{I}$ or set $\mathcal{T} \backslash\left(-\infty, s_{1}\right]$ if $s_{2} \notin \mathcal{I}$. It should be mentioned here that assumptions 2$)-4$ ) of theorem 5.3 supply the existence and uniqueness of the function $x_{2}(t)$ satisfying equation (1.1), starting at either point $\left(s_{1}, x_{1}+I_{1}\left(x_{1}\right)\right)$ (value $x_{1}$ is finite) or point $\left(s_{1}, \infty\right)$ (value $x_{1}=\infty$ ) and ending at either point $\left(s_{2}, x_{2}\right)$ (value $x_{2}$ is finite) or point $\left(s_{2}, \infty\right)$ (value $x_{2}=\infty$ ).

If $s_{2} \notin \mathcal{I}$, then the solution $x_{2}(t)$ is defined on set $\mathcal{T} \cap\left(-\infty, s_{1}\right]$, hence, the solution $x^{*}(t)$ composed on functions $x_{1}(t), x_{2}(t)$ is defined on the whole set $\mathcal{T}$. 
If $s_{2} \in \mathcal{I}$, then the solution to the problem (1.1), (1.2) is constructed on the set $\left(-\infty, s_{2}\right] \cap \mathcal{T}$.

Based on the same arguments, it is easy to see that if the solution $x=x^{*}(t)$ to the problem $(1.1),(1.2)$ is found on interval $\mathcal{T} \cap\left(-\infty, s_{k}\right]$, then it can be extended on interval $\mathcal{T} \cap\left(-\infty, s_{k+1}\right]$ under assumption $s_{k+1} \in \mathcal{I}$, otherwise the solution obviously exists on the whole set $\mathcal{T}$.

Thus, the solution to the problem (1.1), (1.2) can be constructed on the whole set $\mathcal{T}$, which completes the proof of the theorem.

Let us introduce the notion of a piecewise smooth solution.

Definition 5.1. A function $x=x(t)$ is called a piecewise smooth solution to the problem (1.1), (1.2) if it is a solution to the problem (1.1), (1.2) in the sense of definition 2.5 for all $t \in \mathcal{T}$ excepting values, coinciding with $t$-coordinates of critical points where its continuation and smoothness properties may be not satisfied.

The above definition allows us to study a case when the set $\mathcal{T}$ contains a critical point of the equation (1.1) such that its $t$-coordinate coincides with a moment of impulsive effects.

Let us consider sets $\mathcal{T}_{m}^{c r}=\left(\tau_{m}, \tau_{m+1}\right), m \in B$. Here, as before, $\tau_{m}$ are $t$ coordinates of critical points of the equation (1.1), which are ordered with indexes $m$, i.e., $\tau_{m}<\tau_{m+1}$ for all $m, m+1 \in B$ and there exists $\delta_{1}>0$ such that $\tau_{m+1}-\tau_{m}>\delta_{1}$. For the given case by analogy we can formulate a statement summarizing theorems 5.1-5.3.

Theorem 5.4. Let the set $\mathcal{T} \times \overline{\mathbb{R}}^{1}$ contain critical points of the equation (1.1). Then the problem (1.1), (1.2) has a piecewise smooth solution defined on the set $\mathcal{T} \backslash\left\{\tau_{m}, m \in\right.$ B\} iff:

(1) there is a point $(\bar{t}, \bar{x}) \in \mathcal{L}$, a non-critical one of the equation (1.1) such that $\bar{t}<s_{1} \in \mathcal{I}$

(2) there are points $x_{n} \in \overline{\mathbb{R}}^{1}, n \in \mathcal{N}$ such that:

a) $\left\{\left(s_{n}, x_{n}\right), n \in \mathcal{N}\right\} \subset \mathcal{L}$;

b) condition i) or ii) of theorem 3.1 holds;

(3) for any $n \in \mathcal{N} \cup\{0\}$ there exists a set $G_{n} \subset \mathcal{T} \times \overline{\mathbb{R}}^{1}$ such that:

a) set $G_{0}$ is connected and includes points $(\bar{t}, \bar{x})$ and $\left(s_{1}, x_{1}\right)$;

b) for any $n \in \mathcal{N}$ set $G_{n}$ is connected and includes points $\left(s_{n}, x_{n}+I_{n}\left(x_{n}\right)\right)$, $\left(s_{n+1}, x_{n+1}\right)$ provided that $s_{n+1} \in \mathcal{I}$;

(4) for all $n \in \mathcal{N}$ and any $t_{*} \in\left(s_{n}, s_{n+1}\right) \backslash\left\{\tau_{m}: m \in B\right\}$ the equation $g\left(t_{*}, x\right)=0$ has the only solution $x=x_{*}$ such that $\left(t_{*}, x_{*}\right) \in G_{n}$; 
(5) for any $m \in B$ interval $\left(\tau_{m}, \tau_{m+1}\right)$ contains at least one point $t^{*}$ such that the equation $g\left(t^{*}, x\right)=0$ has a solution.

The proof of the theorem is similar to that of theorem $5.1-5.3$ above and therefore it is not given here.

\section{Sufficient conditions of the existence of a periodic solution}

Let us study the existence of a solution to the problem (1.1), (1.2) when function $g(t, x)$ is $T$-periodic with respect to time variable $t$. If a solution to the problem (1.1), (1.2) exists on an interval length of which is more than $T$, then under certain additional conditions solution to the problem under consideration can be continued on the whole set $\mathcal{T}$.

Sufficient conditions of the existence of a periodic solution (a piecewise continuous solution) to the problem (1.1), (1.2) are given by the following theorem.

Theorem 6.1. Let us assume that the following conditions hold:

1) function $g(t, x)$ is $T$-periodic with respect to variable $t$;

2) values of impulsive effects are constants, i.e., $I_{k}(x)=I_{k}$, where $I_{k} \in \mathbb{R}^{1}, k \in \mathcal{N}$;

3) the condition of periodicity of impulsive effects takes place, i.e., there is such a natural $m \in$ that for all $k, k+m \in \mathcal{N}: I_{k+m}=I_{k}, s_{k+m}=s_{k}+T$;

4) problem (1.1), (1.2) has a solution (a piecewise continuous solution) $\varphi(t)$ defined on interval $[\alpha, \beta]$, where $\beta-\alpha=T$ and $\varphi(\alpha)=\varphi(\beta)$.

Then the problem (1.1), (1.2) has a periodic solution (a piecewise continuous solution) defined on the whole set $\mathcal{T} \cap\left[s_{0}, \infty\right)$, where $s_{0}=\min \left\{s_{n}, n \in \mathcal{N}\right\}$.

Proof. As function $\varphi(t), t \in[\alpha, \beta]$, is a solution to the problem (1.1), (1.2), we put $\Phi(t)=\varphi(t-n T)$ for all $t \in[\alpha+n T, \beta+n T] \cap \mathcal{T} \cap\left[s_{0}, \infty\right), n \in \mathbb{Z}$.

Due to definition, function $\Phi(t)$ is defined on the whole set $\mathcal{T}$ and is $T$-periodic in variable $t$. Let us show that $\Phi(t)$ satisfies the equation (1.1). Indeed, we have $g(t+n T, \Phi(t+n T))=g(t, \varphi(t))=0$ for all $t \in[\alpha, \beta] \cap\left[s_{0}, \infty\right)$.

As $\varphi(\alpha)=\varphi(\beta)$ then $\Phi(\alpha)=\Phi(\alpha+s T)$ for any $s \in \mathcal{N}$.

Let us verify whether or not function $\Phi(t)$ satisfies the condition of impulsive effects (1.2). From assumption 3) of theorem 6.1 it follows that for any moment of impulsive effects $s_{k} \in \mathcal{I}$ there is a point $s^{*} \in[\alpha, \beta]$ such that $s^{*}=s_{k}+n_{k} m T$ where $n_{k}$ is some integer and additionally $I_{k}=I_{k+n_{k} m}$.

Then in accordance with construction of function $\Phi(t)$ we get:

$$
\begin{gathered}
I_{k}=\Phi\left(s_{k}+0\right)-\Phi\left(s_{k}-0\right)=\Phi\left(s^{*}-n_{k} m T+0\right)-\Phi\left(s^{*}-n_{k} m T-0\right)= \\
=\varphi\left(s^{*}+0\right)-\varphi\left(s^{*}-0\right)=I_{k+n_{k} m} .
\end{gathered}
$$

Thus theorem 6.1 is proved. 


\section{REFERENCES}

[1] Myshkys A.D. and Samoilenko A.M.: Systems with pushes at the given moments of time, Mathem. sbornik. 74(2), (1967), 202-208. (in Russian)

[2] Samoilenko A.M. and Perestyuk N.A.: Differential equations with the impulse effects, Vyshch. shkola. Golovnoe izd-vo, 1987. (in Russian).

[3] Samoilenko A.M. and Perestyuk N.A.: Impulsive differential equations, World Scientific Series on Nonlinear Sciences. Ser. A. 14, World Scientific, Singapore - New Jersey - London - Hong-Kong, 1995.

[4] Lakshmikantham V., Bainov D.D. and Simeonov P. S.: Theory of impulsive differential equations, World Scientific, Singapore, 1989.

[5] Samoylenko V.Hr. and Yelgondyev K.K.: On periodic solutions to linear differential equations with impulse effect, Ukrainian Mathematical Journ., 49(1), (1997), 141-148. (in Russian)

[6] Perestyuk N.A., Samoylenko V.Hr. and Yelgondyev K.K.: Sequence mapping and periodic solutions to the differential equations with impulsive effects, Nonlinear oscilations, 1(1), (1998), 44-50. (in Russian)

[7] Samoilenko A.M., Samoylenko V.Hr. and Sobchuk V.V.: On periodic solutions to the equation of nonlinear oscillator with impulse effect, Ukrainian Mathematical Journ., 51(6), (1999), 827-834. (in Ukrainian)

[8] Perestyuk M.O. and Lisovska V.P.: Study of periodical solution of weakly nonlinear and singularly perturbed impulse systems, Nonlinear differential systems and its application: Zb. nauk. pr. / AS of Ukraine, Institute of mathematics, (1992), 141-150. (in Ukrainian)

[9] Samoylenko V.Hr. and Kaplun Yu.I.: The equation $g(t, x)=0$ : existence and continuation of its solutions, Ukrainian Mathematical Journ., 53(3), (2001), 272-282. (in Ukrainian)

[10] Samoylenko V.Hr. and Kaplun Yu.I.: On global solutions to the functional equation, Differential Equations, 6(11), (2000), 1578-1583. (in Russian) 